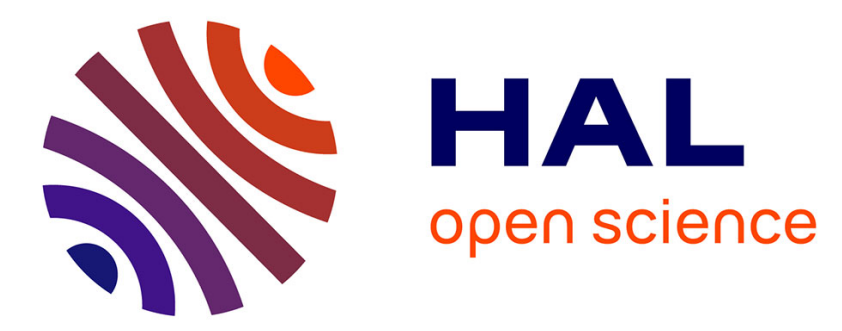

\title{
L'organisation de la lutte contre la cécidomyie des fleurs de luzerne (Contarinia medicaginis Kieff.) : contribution des travaux réalisés en conditions agronomiques
}

René Bournoville, Alain Delaude, Pierre Cantot

\section{- To cite this version:}

René Bournoville, Alain Delaude, Pierre Cantot. L'organisation de la lutte contre la cécidomyie des fleurs de luzerne (Contarinia medicaginis Kieff.) : contribution des travaux réalisés en conditions agronomiques. Agronomie, 1986, 6 (4), pp.393-400. hal-00884890

\section{HAL Id: hal-00884890 https://hal.science/hal-00884890}

Submitted on 1 Jan 1986

HAL is a multi-disciplinary open access archive for the deposit and dissemination of scientific research documents, whether they are published or not. The documents may come from teaching and research institutions in France or abroad, or from public or private research centers.
L'archive ouverte pluridisciplinaire HAL, est destinée au dépôt et à la diffusion de documents scientifiques de niveau recherche, publiés ou non, émanant des établissements d'enseignement et de recherche français ou étrangers, des laboratoires publics ou privés. 


\title{
L'organisation de la lutte contre la cécidomyie des fleurs de luzerne (Contarinia medicaginis Kieff.) : contribution des travaux réalisés en conditions agronomiques
}

\author{
René BOURNOVILLE \& Alain DELAUDE $\left({ }^{*}\right)$ \\ avec la collaboration technique de Pierre CANTOT \\ I.N.R.A., Laboratoire de Zoologie, F 86600 Lusignan \\ (*) Centre Technique de la Fédération Nationale des Agriculteurs Multiplicateurs de Semences, 9, rue des \\ Fleurs, F 49000 Angers
}

La cécidomyie des fleurs de la luzerne (Contarinia medicaginis Kieff., Dipt. Cecidomyidae) est l'insecte le plus nuisible à la production de graines de luzerne en France comme dans une grande partie de l'Europe. Des expérimentations ont été entreprises afin de disposer, dans les conditions de la pratique agricole, de méthodes fiables de lutte contre ce ravageur. Les essais d'élevages de larves hivernantes n'ont pas permis de mettre au point un système d'avertissement. Les apparitions d'adultes sont en effet décalées par rapport à l'abondance des captures réalisées par piégeage. L'utilisation de pièges colorés à eau ne permet pas non plus d'intervention efficace dans la mesure où la ponte des femelles a pu précéder leur capture. Du point de vue pratique, cette dernière technique ne peut d'ailleurs concerner qu'une parcelle déterminée en raison des importantes variations qui ont pu être constatées entre les résultats acquis dans les champs d'une même zone. On a donc fait porter l'expérimentation sur la protection préventive de très jeunes boutons floraux réceptifs à la ponte. La première intervention contre la cécidomyie des fleurs doit avoir lieu lorsqu'une inflorescence est différenciée pour 2 tiges principales, ce que l'on établit sur un échantillon de 25 tiges.

Les essais d'efficacité de produits ont montré que, parmi les pyréthrinoïdes, la deltaméthrine et le fenvalérate ont une efficacité très supérieure à celle des produits classiques recommandés jusqu'ici pour lutter contre la cécidomyie. Les autres possibilités de lutte sont évoquées dans la discussion : façons culturales, traitements du sol, moyens biologiques.

Mots clés additionnels : Piégeage, avertissements agricoles, phénologie, pyréthrinoïdes, traitements insecticides.

Contribution of field experiments to the control of the lucerne flower gall midge Contarinia medicaginis Kieff.

Field experiments were carried out mainly in West and South West France on the flower gall midge, Contarinia medicaginis Kieft. (Dipt. Cecidomyidae), an important pest of lucerne grown for seed production in Europe. Several methods were applied to estimate the abundance of adults and to provide warnings : breeding of wintering larvae or trapping of newly emerging adults in the field. The results of these two methods, applied in the same zone, were not similar and no practical applications in determining the date of an insecticide treatment could be drawn from the results. Nevertheless, trapping showed that females are mainly captured at the upper level of vegetation and males at ground level.

We reconsidered the protection of the small buds in which the midge lays its eggs. Study of the phenology of lucerne fields showed that the crop has to be protected very early. The insecticide treatment has to be applied as soon as 1 inflorescence is differentiated on 2 main stems (the sample being composed of 25 plants). We compared the efficacy of some pyrethroids to the activity of insecticides harmless to pollinators. Deltamethrine and fenvalerate were very active in controlling the gall midge. The treatment has to be repeated 12 days later. The discussion deals with other potential methods of controlling $C$. medicaginis.

Additional key words : Trapping, spray advice, phenology, pyrethroids, insecticide treatments. 


\section{INTRODUCTION}

L'organisation de la lutte contre la cécidomyie des fleurs de la luzerne Contarinia medicaginis Kieff., pose des problèmes difficiles à résoudre en Europe. Dans une récente enquête sur les ravageurs de la production de graines de luzerne (BOURNOvILLE et al., 1984), cet insecte est cité au $1^{\text {er }}$ rang de nuisibilité dans 11 pays sur 13 . Il n'y a que dans la péninsule ibérique où cet insecte n'est pas mentionné parmi les 3 ravageurs majeurs de la luzerne porte-graines. L'appréciation de la gravité des attaques de cette cécidomyie est certes liée au côté spectaculaire de ses manifestations par les galles induites par les larves de cet insecte, mais l'intensité des dégâts peut varier annuellement et localement. Ainsi en France, CAIRASCHI (1973), dans une étude réalisée dans le Lauraguais, considère que l'insecte le plus à craindre pour la production de semences de luzerne est la cécidomyie des pousses (Dasyneura ignorata Wacht.). STREBLER (1968) souligne pour sa part que $C$. medicaginis n'est à mettre en cause que pour le quart des pertes du potentiel floral de la luzerne. Pour situer les faits, on signalera que, dans un ensemble de 6 essais de lutte chimique réalisés en 1977 dans le Sud-Ouest, les parcelles témoins comportaient 8 galles par inflorescence, ce qui représente de 33 à 50 p. 100 du potentiel floral.

Malgré certaines ambiguïtés tenant soit à l'interférence de nombreux facteurs responsables de la chute des fleurs de luzerne, soit à la méthodologie d'appréciation des dégâts de la cécidomyie, il devenait important à partir de 1975 de proposer une méthode fiable d'intervention contre cet insecte, et cela d'autant plus que les erreurs dans l'application des traitements se faisaient plus fréquentes. C'est ainsi que, lors d'une enquête, BOURNOVILLE \& BOURDONCLE (1978) notent que 45 p. 100 des traitements réalisés contre la cécidomyie des fleurs sont mal situés compte tenu du stade du végétal à protéger. Les interventions trop tardives faisaient courir de grands risques à la faune des pollinisateurs, puisque, dans 10 p. 100 des cas, des produits toxiques à leur égard étaient utilisés en phase florale. Cet état de fait ne provenait pas d'un manque d'informations sur la biologie de l'insecte. On citera à ce propos, les publications en France de CouTin (1962) et de STREBLER $(1968,1975)$. Ces auteurs insistent notamment sur les liens étroits entre la plante et l'insecte et précisent le cycle biologique dans les conditions françaises.

Un rappel des principaux résultats publiés sur ce sujet est nécessaire à la présentation de nos travaux. La cécidomyie des fleurs de luzerne pond ses œufs dans des boutons verts. C'est à ce stade que la structure des pièces florales permet à l'insecte d'insérer ses œufs (COUTIN, 1962). STREBLER distingue 7 stades d'inflorescences et 7 stades floraux. Les stades réceptifs à la ponte de $C$. medicaginis sont les 3 premiers, le stade le plus sensible étant constitué par l'inflorescence verte dont le pédoncule atteint $1 \mathrm{~cm}$. Le cycle de l'insecte comporte une génération hivernante dont les larves après leur diapause se transforment en pupes au printemps. Les adultes apparaissent puis s'accouplent ; leur longévité est faible ( $5 \mathrm{j}$ au maximum). La fécondité moyenne des femelles est de 65 œufs. Elles peuvent les déposer à raison de 3 ou 4 par bouton ; des pontes multiples expliquent la variation du nombre de larves par galle ( 1 à 20 ). Après quelques jours de développement embryonnaire ( 3 à $4 \mathrm{j}$ à $20^{\circ} \mathrm{C}$ ), la durée du développement larvaire varie de 3 à 6 semaines. Les larves induisent la transformation des fleurs en galles, dont elles sortent à leur complet développement pour réaliser dans le sol un cocon de nymphose ovoüde. Après 2 semaines, de nouveaux adultes de $1^{\mathrm{re}}$ génération apparaissent et sont à l'origine d'une $2^{\mathrm{e}}$ génération. Lors de ces 2 générations, une proportion croissante de larves reste en diapause dans le sol jusqu'à l'année suivante.

Devant l'insuffisance des résultats de la lutte contre la cécidomyie des fleurs, nous avons réalisé des expérimentations sur le terrain afin de vérifier les résultats acquis au laboratoire. Notre investigation a porté sur des techniques permettant de détecter la présence des adultes contre lesquels la lutte chimique est dirigée. Nous avons par ailleurs précisé la liaison de l'insecte avec la phénologie du peuplement végétal, ce qui complète les éléments publiés sur le stade du végétal infesté, afin d'assurer la protection efficace de la culture. Nous avons précisé la méthodologie de l'estimation des dégâts dus à $C$. medicaginis qui était peu nette dans la bibliographie. Notre recherche a enfin porté sur le choix de nouvelles matières actives contre la cécidomyie. Ces divers points ont amené à redéfinir la stratégie de la lutte chimique contre $C$. medicaginis pour laquelle la disponibilité des pyréthrinoïdes de synthèse a offert de nouvelles possibilités.

\section{MATÉRIEL ET MÉTHODE}

\section{A. Estimation des populations imaginales}

STREBLER (1975) a étudié les techniques disponibles pour apprécier la présence et l'abondance des adultes. Il s'agit de l'élevage des cocons hivernants en conditions extérieures afin de connaître l'époque d'apparition des imagos et du piégeage de ces derniers au champ.

\section{Elevage des hivernants}

A la fin de l'été, dans des champs infestés par la cécidomyie, on recueille des galles qui commencent à se décolorer mais qui ne sont pas encore fanées ou brunies. A ce stade d'évolution, elles renferment encore les larves. On dépose 100 de ces galles sur un tamis à la surface d'un godet de terre cuite rempli d'un mélange de terre et de terreau. Ce godet est recouvert d'un pot transparent. Les galles sont retirées après une dizaine de jours parce qu'elles pourrissent et que les larves se sont enfouies dans la terre du godet. STREBLER conseille un arrosage régulier $(3 \mathrm{ml}$ par godet de $5 \mathrm{~cm}$ de diamètre) afin de fournir une humidité sans excès. Les godets sont groupés dans un bac rempli de sable maintenu également humide. Pour apprécier l'apparition des hivernants l'année suivant la récolte des galles, on conserve cet ensemble dans un lieu soumis aux températures extérieures. La surveillance des émergences doit être continuelle à partir du printemps. La population de ces hivernants permet de 
déterminer le début du vol au printemps suivant, même si elle ne représente pas toute l'hétérogénéité de la population hivernante naturelle (STREBLER, 1975). Par convention, cet auteur appelle « vols » les relevés rassemblant au moins 10 individus par semaine dans un élevage unitaire de 100 galles initiales.

\section{Le piégeage d'adultes}

Malgré des difficultés d'interprétation des résultats, STREBLER (1975) signale que le piégeage des cécidomyies au moyen de bac ou d'assiettes contenant de l'eau et un mouillant peut suppléer à l'absence d'élevage des hivernants. Il signale que les couleurs jaune et bleu sont attractives.

Dans un essai préliminaire, nous avons disposé des assiettes de $20 \mathrm{~cm}$ de diamètre peintes en couleur « jaune bouton d'or - Ripolin 514 » dans une parcelle. Cinq assiettes sont disposées sur le sol à environ $10 \mathrm{~m}$ de distance l'une de l'autre, les 5 autres étant placées sur des supports permettant de déplacer le piège selon le niveau supérieur de la végétation. Les assiettes sont remplies d'eau et de mouillant. Elles sont relevées 3 fois par semaine. Les insectes sont conservés dans de l'alcool avant leur examen au laboratoire. Pour cela, les cécidomyies sont montées entre lame et lamelle une à une dans un milieu d'observation afin d'être examinées au microscope. Sur l'ensemble des 2 périodes de piégeage, ce sont plus de 12000 cécidomyies qui ont été ainsi observées une à une (tabl. 1). Les caractères de détermination de $C$. medicaginis tiennent à la nervation alaire, à l'extrémité abdominale où se trouvent les pièces génitales et l'ovipositeur et à la morphologie antennaire. Cette étude nous a permis de montrer que la femelle de C. medicaginis est piégée à la partie haute de la végétation, tandis que les mâles se font piéger au niveau du sol. C'est donc au niveau supérieur de la végétation qu'ont été placés tous les pièges lors des expérimentations ultérieures.

\section{B. Estimation de la phénologie du peuplement végé- tal}

Les travaux de STREBLER (1968) ont particulièrement bien mis en évidence le stade de la fleur ou de l'inflorescence sensible à la ponte de $C$. medicaginis.
Cependant, les références manquent pour caractériser le stade d'une tige qui comporte plusieurs inflorescences et à fortiori celui du peuplement végétal qui, dans le cas de la luzerne, est très hétérogène. On a donc, après de nombreux essais, relié les populations de l'insecte aux caractéristiques suivantes de la luzernière :

- la courbe de floraison. Elle est établie en lançant au hasard dans la végétation un cerceau de $50 \mathrm{~cm}$ de diamètre. Dans le cercle ainsi délimité, on compte les inflorescences présentant au moins une fleur épanouie. On réalise 5 répétitions de ce comptage ;

- le nombre d'entre-nœuds floraux. La disposition des inflorescences chez la luzerne est du type « inflorescence indéfinie » en grappe. Des essais antérieurs ont montré que l'échantillon des tiges est représentatif en choisissant, sur des portions successives de $20 \mathrm{~cm}$ des lignes de semis, les 3 tiges suivant les 2 tiges les plus grandes ( $\mathrm{J}$. HACQUET, comm. pers.). On repère ainsi 25 à 30 tiges sur lesquelles est noté le nombre de nœuds floraux de la tige principale, c'est-à-dire d'une tige partant du sol, ce qui exclut donc les ramifications.

\section{Les essais de traitement insecticide}

De nombreux essais ont été réalisés sur le terrain. Le dispositif utilisé est du type essai bloc à répétitions ; la parcelle élémentaire mesure $4 \times 10 \mathrm{~m}=40 \mathrm{~m}^{2}$ de surface. Une bande d'isolement de $1 \mathrm{~m}$ de large sépare les parcelles. L'essai est placé en conditions agronomiques dans une zone de parcelles de production qui ne reçoit pas de traitement insecticide. Le reste du champ subit les interventions jugées nécessaires par le producteur. De 1977 à 1981, le nombre d'essais mis en place annuellement a été respectivement de $4,12,6,7$ et 5 .

\section{Quantification des dégâts de la cécidomyie}

Dans les premiers essais comparatifs de traitements insecticides, l'importance des dégâts est estimée par des notations visuelles de dégâts sur chaque parcelle

TABLEAU 1

Piégeage de C. medicaginis avec des récipients colorés (I.N.R.A., Tarn).

Trapping of C. medicaginis with coloured water plates (I.N.R.A., Tarn).

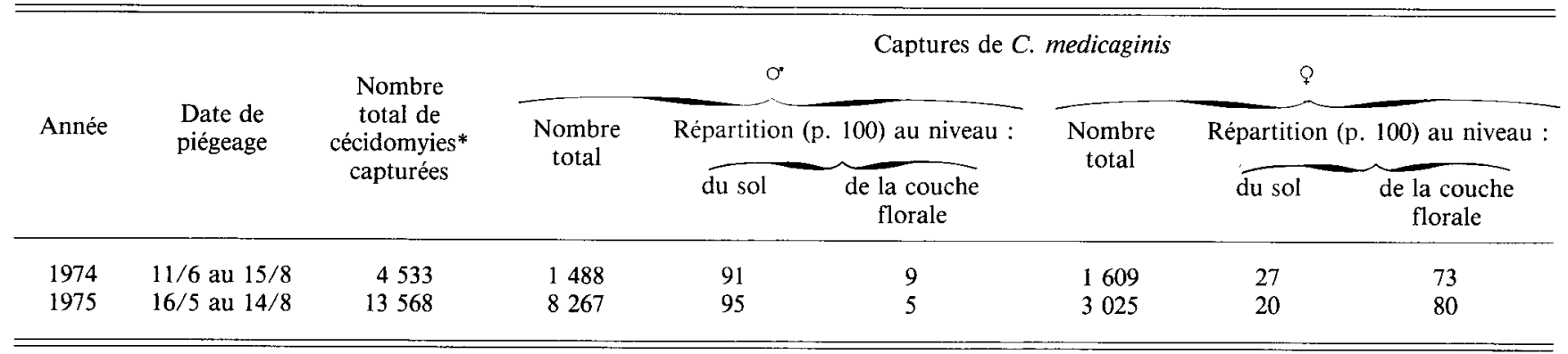

* Ensemble des individus de la famille. 
comparativement aux bandes témoins non traitées. Le dégât observé dans ce témoin reçoit la note 10 , l'absence totale de dégâts étant notée 0 . En cas d'infestations élevées, on a pu vérifier que ces notations sont représentatives des rendements parcellaires, mais ce type de notation est délicat à réaliser en cas de faible infestation. On a donc compté le nombre de galles sur 25 tiges principales. Après avoir ramené ce nombre à des classes de notation (tabl. 2), une corrélation très hautement significative de cette notation avec le rendement $(\mathrm{r}=-0,91)$ a pu être établie (Bournoville \& Bourdoncle, 1978). Cependant, il s'agit là d'un échantillonnage limité où le choix des tiges peut introduire un biais important.

Une autre possibilité est l'évaluation du nombre de galles par unité de surface. Pour cela, lors de l'établissement de la courbe de floraison, on compte les inflorescences portant des galles $(=$ a) puis, sur un échantillon de 20 inflorescences, on évalue le nombre moyen de galles par inflorescence $(=b)$. Le nombre moyen de galles par unité de surface est le produit ab. Les variations importantes du peuplement végétal entre les luzernières d'âge différent rendent cependant difficiles, avec ce système, les comparaisons entre les zones d'un même champ et entre divers lieux. Les dégâts sont donc finalement exprimés par le nombre de galles par inflorescence. Celui-ci est obtenu en faisant le rapport du nombre total de galles sur le nombre d'inflorescences isolées lors des lancers de cerceau. Le gain de précision obtenu par rapport au comptage précédent apparaît nettement lorsqu'on compare les coefficients de variation qui sont diminués de moitié.

\section{RÉSULTATS}

\section{A. Populations imaginales}

L'expérimentation a disposé chaque année d'un seul essai d'élevage de larves hivernantes. Certains échecs initiaux ont pu être attribués à une mauvaise appréciation par l'expérimentateur du stade des galles à prélever, ce qui peut impliquer que les larves avaient déjà quitté les galles lors de leur prélèvement. On a donc cherché à estimer dès le début de l'élevage le nombre de larves présentes par galle en plaçant un lot témoin de galles au-dessus d'un récipient rempli d'eau. En moyenne, cet effectif se situe aux environs de 1 larve par galle (tabl. 3). Les résultats finals de l'élevage montrent les importantes pertes d'effectifs larvaires lors de l'élevage hivernal puisque ce n'est guère que dans $1 / 4$ des cas que l'on retrouve des effectifs d'adultes comparables à ceux des larves initiales.

La mise en ouvre par divers techniciens (FNAMS, SPV) de la technique du piégeage avec des récipients colorés a rapidement montré les limites de son utilisation pratique pour l'organisation de la lutte : en effet, il n'est pas toujours possible de mettre en évidence des générations bien tranchées, car l'étalement des vols va souvent de pair avec l'intensité des dégâts.

Les résultats d'une expérimentation conduite en 1978 sur 24 champs dans 4 départements ont montré des écarts de plusieurs jours entre les captures maximales. Au sein d'une même zone géographique, on a des intensités et des dates de pics de vols assez diver-

TABLEAU 2

Notation des dégâts de $\mathrm{C}$. medicaginis sur 25 tiges principales. Scoring of damage by $\mathrm{C}$. medicaginis on 25 main stems.

\begin{tabular}{|c|c|c|c|c|c|c|c|c|c|c|}
\hline Nombre de galles & $<100$ & $\begin{array}{r}100 \\
\grave{a} \\
<200\end{array}$ & $\begin{array}{r}200 \\
\text { à } \\
<300\end{array}$ & $\begin{array}{r}300 \\
\dot{a} \\
<400\end{array}$ & $\begin{array}{c}400 \\
\text { à } \\
<500\end{array}$ & $\begin{array}{c}500 \\
\grave{a} \\
<600\end{array}$ & $\begin{array}{c}600 \\
\text { à } \\
<700\end{array}$ & $\begin{array}{r}700 \\
\text { à } \\
<800\end{array}$ & $\begin{array}{c}800 \\
\grave{a} \\
<900\end{array}$ & $\begin{array}{r}900 \\
\text { à } \\
<1000\end{array}$ \\
\hline Notes & 1 & 2 & 3 & 4 & 5 & 6 & 7 & 8 & 9 & 10 \\
\hline
\end{tabular}

TABLEAU 3

Elevage de larves hivernantes.

Breeding of wintering larvae.

\begin{tabular}{|c|c|c|c|c|}
\hline Année & Organisme & $\begin{array}{c}\text { Lieu } \\
\text { (Département) }\end{array}$ & $\begin{array}{l}\text { Nbre de larves initiales } \\
\text { pour } 100 \text { galles }\end{array}$ & $\begin{array}{l}\text { Nbre d'adultes obtenus } \\
\text { pour } 100 \text { galles }\end{array}$ \\
\hline $1973-74$ & I.N.R.A. & Lusignan (86) & 90 & 14 \\
\hline \multirow[t]{2}{*}{$1975-76$} & S.P.V. & Toulouse (31) & $?$ & 43 \\
\hline & I.N.R.A. & Lusignan (86) & 150 & 36 \\
\hline $1976-77$ & S.P.V. & Toulouse (31) & $?$ & 3 \\
\hline $1977-78$ & I.N.R.A. & Lusignan (86) & 120 & 88 \\
\hline $1979-80$ & I.N.R.A. & Lusignan (86) & 120 & 9 \\
\hline $1980-81$ & I.N.R.A. & Lusignan (86) & 90 & 93 \\
\hline
\end{tabular}


ses. Comme l'a souligné STREBLER (1975), cela fait bien ressortir le caractère aléatoire de ces prévisions puisque les conditions extérieures au moment des éclosions ont un effet important sur les vols. Enfin, le caractère fastidieux de ce type de relevé, qui demande des passages réguliers et un important travail de tri et de détermination, ne saurait être négligé.

A quelques reprises, les 2 techniques ont pu être comparées dans un même lieu. Elles fournissent des résultats souvent différents (fig. 1). Cela est d'autant plus vrai que les conditions climatiques sont plus contrastées et qu'elles favorisent la sortie brusque des cécidomyies au champ.

\section{B. La lutte insecticide fondée sur la protection du stade du végétal sensible à la ponte}

Les difficultés de mise en pratique ou l'inefficacité des techniques précédentes nous ont conduits à réexaminer les possibilités de la protection du végétal durant sa phase réceptive à la ponte. Nous avons fait porter nos travaux sur l'efficacité de diverses matières actives et la définition du moment d'intervention.

\section{Le stade du peuplement végétal lors du $1^{\text {er }}$ traite- ment}

Dans les premiers essais, le $1^{\text {er }}$ traitement a été fixé au moment où l'échantillon des tiges principales présente 3 inflorescences différenciées. Dans l'ensemble de ce texte, on désigne par «inflorescence différenciée » celle dont le pédoncule est visible pour l'expéri- mentateur. Les nombreux comptages réalisés prouvent que, lorsque des cécidomyies sont présentes sur la culture avant ce stade, de fortes infestations peucul se manifester : celles-ci s'expliquent assez bien si l'on considère que, pour une tige, en plus des 3 inflorescences différenciées, on trouve dans le bourgeon terminal un nombre variable de très jeunes inflorescences (de l'ordre de 3 à 5) dont une majorité de boutons peut recevoir les pontes. Il s'avère donc qu'environ 6 à 8 niveaux de floraison peuvent être infestés, ce qui peut représenter jusqu'à la moitié du potentiel de fructification. Par la suite, on a donc avancé l'époque du traitement à 1 inflorescence différenciée par tige principale. Un plus grand nombre de niveaux de floraison ont été ainsi protégés ce qui ressort de la différence significative de galles présentes dans le témoin et dans les lots traités $20 \mathrm{j}$ après l'intervention. Le manque de signification $10 \mathrm{j}$ après le traitement nous a poussés à avancer encore ce moment du $1^{\text {er }}$ traitement à 1 inflorescence différenciée pour 2 tiges. Même si on constate encore quelques dégâts, les pertes sont minimes car la floraison est efficacement protégée.

\section{L'action des produits}

Jusqu'à une date récente, le toxaphène, l'endosulfan, la phosalone, le bromophos et le dialiphos étaient les seuls produits utilisables contre la cécidomyie en période florale. Leur action est incomplète notamment par manque de rémanence. Si la protection est suffisante par beau temps et avec de faibles infestations d'insectes, elle devient médiocre à très médiocre lors-

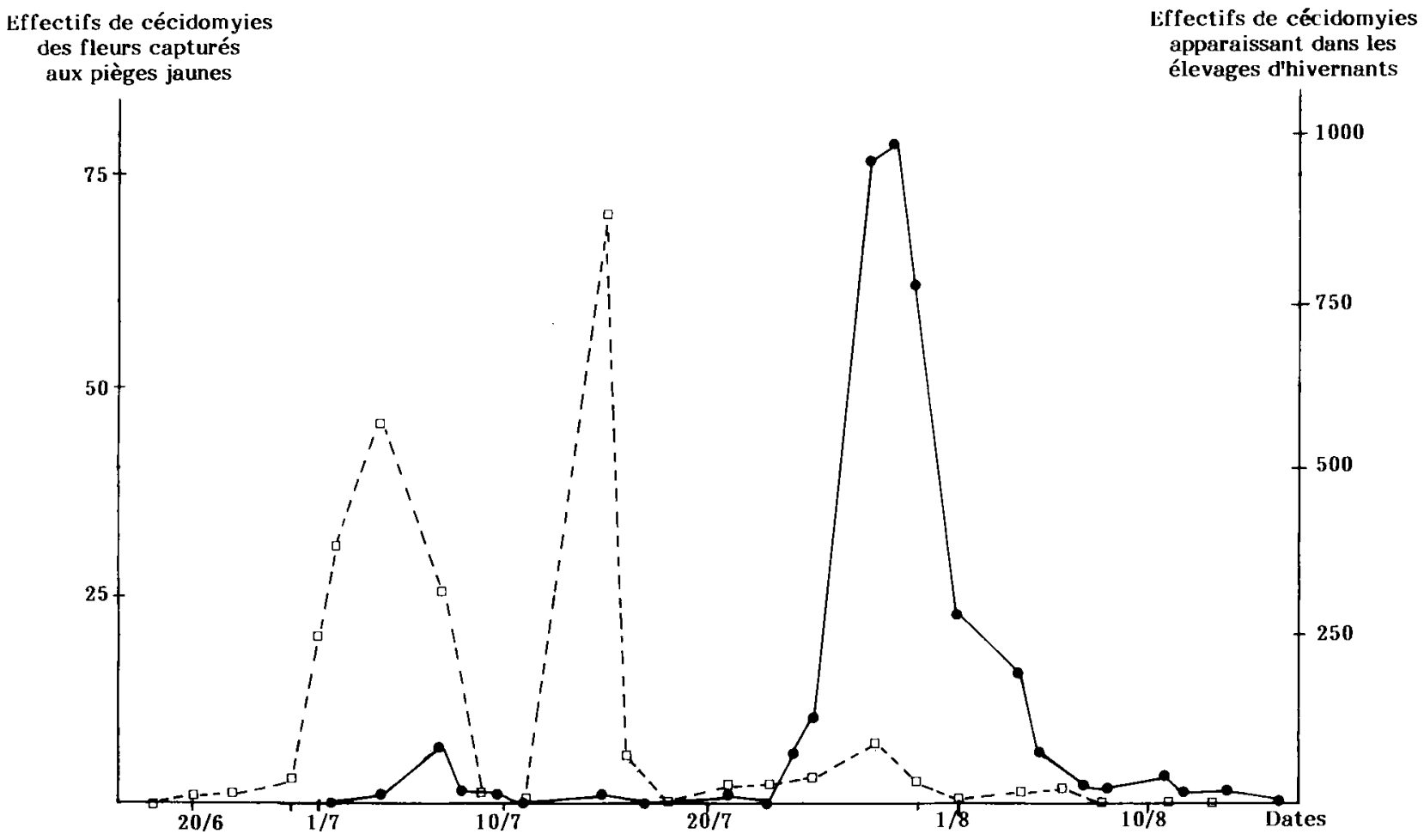

Figure 1

Comparaison du piégeage au champ des adultes de C . medicaginis (traits pointillés, ordonnées de gauche) et de l'émergence des hivernants (traits pleins, ordonnées de droite). (Lusignan, I.N.R.A., 1980.)

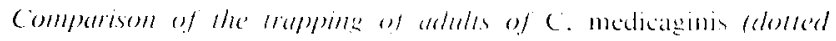
line, left $Y$-axis) in the field and of the emergence of wintering adults (full line, right Y-axis). (Lusignan, I.N.R.A., I980.) 
que la pluie lessive ces produits. Le tableau 4 résume l'ensemble de 8 essais ayant reçu ces traitements insecticides bien positionnés par rapport à la phénologie. Dans le meilleur des cas, le traitement n'a qu'une efficacité de 25 p. 100 ce qui est très insuffisant.

Dès 1979, les premiers essais réalisés avec les pyréthrinoïdes de synthèse se sont révélés encourageants. Deux matières actives se sont montrées intéressantes : la deltaméthrine et le fenvalérate (tabl. 5). Ce dernier, en raison de son action secondaire très limitée sur le mégachile (DEBRAY et al., 1984), pollinisateur de référence de la luzerne, a reçu une autorisation d'emploi pour le traitement contre la cécidomyie des fleurs de la luzerne. La persistance d'action du fenvalérate implique un $2^{\mathrm{e}}$ traitement $12 \mathrm{à} 15 \mathrm{j}$ après le $1^{\mathrm{er}}$. La rémanence d'ensemble de ces 2 traitements permet de protéger efficacement la partie de la floraison intéressant la production grainière comme le montrent les résultats du tableau $5 \mathrm{~A}$.

\section{La lutte insecticide dirigée sur les jeunes larves de l'insecte présentes dans le bouton floral}

La suppression de la jeune larve par l'action systémique d'un produit serait une autre possibilité de lutte, sous réserve qu'elle intervienne avant toute action déformante des larves. Cette modalité nécessite l'emploi de produits pénétrants ou endothérapiques. Ce type d'action peut être observé lorsque des essais de traitement sont conduits notamment après un vol important d'adultes, ce qui exclut l'action directe des insecticides sur l'adulte. Dans le cas du tableau 6, la deltaméthrine ne se différencie pas de la phosalone, du bromophos, du trichlorfon, du formétanate puisque les traitements sont trop tardifs pour atteindre les adultes alors que l'oxydéméton et le fenthion se classent mieux.

Cette efficacité est très insuffisante et sans commune mesure avec ce que peuvent donner les produits actifs lorsqu'ils sont appliqués sur le végétal avant la ponte de l'adulte.

\section{DISCUSSION}

Dans la pratique, la protection de la luzerne, lors de la phase sensible à la ponte de la cécidomyie, est très satisfaisante si les modalités d'application que nous venons d'analyser sont respectées; néanmoins, il s'agit encore d'une méthode préventive qui peut impliquer des traitements inutiles. D'autres solutions potentielles ont été avancées par divers auteurs.

\section{A. Les techniques culturales}

Des observations réalisées dans le Sud-Ouest (CAIRASCHI, 1973) tendent à conclure qu'un décalage de coïncidence entre l'insecte et la plante hôte peut éviter une bonne part des attaques de $C$. medicaginis. Les cultures dont la production grainière est réalisée sans fauche printanière paraissent échapper aux vols massifs du ravageur. Notre expérience a montré (tabl. 7) qu'il n'est pas rare que de tels types de culture (qu'on
TABLEAU 4

Efficacité de divers insecticides (moyenne de 8 essais - 1978 - FNAMS).

Efficacy of different insecticides (means of 8 tests - 1978 - FNAMS).

\begin{tabular}{lcc}
\hline \hline \multicolumn{1}{c}{ Produits } & $\begin{array}{c}\text { Dose } \\
\text { (g de M.A./ha) }\end{array}$ & $\begin{array}{c}\text { Nombre de galles } \\
\text { pour } \\
100 \text { inflorescences* }\end{array}$ \\
\hline $\begin{array}{l}\text { Phosalone } \\
\text { Dialiphos + endosulfan }\end{array}$ & 1000 & 611 \\
Bromophos & $324+262$ & 692 \\
Toxaphène + trichlorfon & $1350+480$ & 700 \\
Témoin & & 712
\end{tabular}

* Les nombres réunis par un trait ne diffèrent pas significativemen pas entre eux au seuil de 5 p. 100.

TABLEAU $5 \mathrm{~A}$

Efficacité de 2 pyréthrinoïdes contre $\mathrm{C}$. medicaginis : S.P.V., Poitiers, 1981.

Efficacy of 2 pyrethroids against $\mathrm{C}$. medicaginis : S.P.V., Poitiers, 1981.

Modalités

(Matière active et dose en $\mathrm{g} / \mathrm{ha}$ )

Traitements le jour :

Nombre de galles pour 100 inflorescences* au jour :

\begin{tabular}{ccrc}
$J$ & $J+14$ & $J+24$ & $J+34$ \\
\hline$F(50 \mathrm{~g})$ & $\mathrm{P}(1000 \mathrm{~g})$ & $88 \mathrm{a}$ & $53 \mathrm{ab}$ \\
$\mathrm{F}(50 \mathrm{~g})$ & & $89 \mathrm{a}$ & $101 \mathrm{bc}$ \\
$\mathrm{F}(30 \mathrm{~g})$ & & $165 \mathrm{a}$ & $220 \mathrm{c}$ \\
$\mathrm{F}(50 \mathrm{~g})$ & $\mathrm{F}(50 \mathrm{~g})$ & $76 \mathrm{a}$ & $19 \mathrm{a}$ \\
$\mathrm{F}(30 \mathrm{~g})$ & $\mathrm{F}(30 \mathrm{~g})$ & $83 \mathrm{a}$ & $28 \mathrm{a}$ \\
$\mathrm{D}(7,5 \mathrm{~g})$ & & $98 \mathrm{a}$ & $215 \mathrm{c}$ \\
Témoin & & $548 \mathrm{~b}$ & $285 \mathrm{c}$ \\
(non traité) & & &
\end{tabular}

$\mathrm{P}=$ phosalone, $\mathrm{D}=$ deltaméthrine, $\mathrm{F}=$ fenvalérate. Traitement unique ou répété.

* Les nombres d'une colonne suivis d'une même lettre ne diffèrent pas entre eux au seuil de 5 p. 100.

TABLEAU 5 B

Efficacité de 2 pyréthrinoïdes contre $\mathrm{C}$. medicaginis : FNAMS, Tarn, 1981.

Efficacy of 2 pyrethroids against $\mathrm{C}$. medicaginis : FNAMS, Tarn, 1981.

\begin{tabular}{cccc}
\hline \hline $\begin{array}{l}\text { Modalités } \\
\text { (Matière active et dose en } \mathrm{g} / \mathrm{ha})\end{array}$ & \multicolumn{2}{c}{$\begin{array}{c}\text { Nombre de galles } \\
\text { pour l00 inflorescences* }\end{array}$} \\
$\begin{array}{c}\text { Traitements le jour : } \\
\mathrm{J}\end{array}$ & $\mathrm{J}+15$ & $\mathrm{~J}+24$ & $\mathrm{~J}+34$ \\
\hline $\mathrm{D}(7,5 \mathrm{~g})$ & $\mathrm{P}(1000 \mathrm{~g})$ & $141 \mathrm{a}$ & $641 \mathrm{bc}$ \\
$\mathrm{D}(7,5 \mathrm{~g})$ & & $167 \mathrm{a}$ & $595 \mathrm{bc}$ \\
$\mathrm{D}(15 \mathrm{~g})$ & & $225 \mathrm{a}$ & $545 \mathrm{bc}$ \\
$\mathrm{D}(7,5 \mathrm{~g})$ & $\mathrm{D}(7,5 \mathrm{~g})$ & $199 \mathrm{a}$ & $159 \mathrm{a}$ \\
$\mathrm{D}(5 \mathrm{~g})$ & $\mathrm{D}(5 \mathrm{~g})$ & $160 \mathrm{a}$ & $307 \mathrm{ab}$ \\
$\mathrm{F}(50 \mathrm{~g})$ & & $159 \mathrm{a}$ & $470 \mathrm{bc}$ \\
Témoin & & $584 \mathrm{~b}$ & $753 \mathrm{c}$ \\
(non traité) & & & \\
\hline
\end{tabular}

$\mathrm{P}=$ phosalone, $\mathrm{D}=$ deltaméthrine, $\mathrm{F}=$ fenvalérate. Traitement unique ou répété.

* Les nombres d'une colonne suivis d'une même lettre ne diffèrent pas entre eux au seuil de 5 p. 100 
Notation des dégâts de la cécidomvie des fleurs dans un essai de lutte insecticide (traitements réalisés après un vol important de cécidomyies) (FNAMS, 1979, Gers).

Scoring of damage by the flower gall midge in an insecticide test

(treatment done after a major flight of midges) (FNAMS, 1979, Gers).

\begin{tabular}{|c|c|c|c|c|c|c|c|}
\hline $\begin{array}{l}\text { Matières } \\
\text { actives } \\
\text { et doses } \\
(\mathrm{g} / \mathrm{ha})\end{array}$ & $\begin{array}{l}\text { Témoin } \\
\text { non traité }\end{array}$ & $\begin{array}{c}\text { Phosalone } \\
1050\end{array}$ & $\begin{array}{c}\text { Bromophos } \\
500\end{array}$ & $\begin{array}{c}\text { Trichlorfon } \\
1200\end{array}$ & $\begin{array}{c}\text { Deltaméthrine } \\
7,5\end{array}$ & $\begin{array}{l}\text { Oxydéméton- } \\
\text { méthyl } \\
500\end{array}$ & $\begin{array}{c}\text { Fenthion } \\
550\end{array}$ \\
\hline Note & 10 & 7,0 & 8,5 & 8,5 & 8,0 & 6,0 & 5,5 \\
\hline
\end{tabular}

\section{TABLEAU 7}

Dégâts de C. medicaginis dans des cultures précoupées $(=p)$ ou non précoupées $(=n p)$ et d'âges différents (I.N.R.A. - Tarn).

Damage by C. medicaginis in "pre-harvested" $(=p)$ and "non pre-harvested" lucerne $(=n p)$ crops of different ages (I.N.R.A. - Tarn).

\begin{tabular}{|c|c|c|}
\hline Age de la culture & $\begin{array}{c}1974 \\
\text { Note (cf. tabl. 2) }\end{array}$ & $\begin{array}{l}1975 \\
\text { Note }\end{array}$ \\
\hline Semis de l'année & $\begin{array}{ll}\text { np } & 3 \\
\text { np } & 4 \\
\text { np } & 3\end{array}$ & $\begin{array}{ll}\text { np } & 9 \\
\text { np } & 5 \\
\text { np } & 10\end{array}$ \\
\hline 1 an & $\begin{array}{ll}\mathrm{p} & 5 \\
\mathrm{p} & 1 \\
\mathrm{p} & 2 \\
\mathrm{p} & 2 \\
\mathrm{p} & 2\end{array}$ & $\begin{array}{ll}\text { p } & 5 \\
\text { np } & 4 \\
\text { np } & 7 \\
\text { p } & 6\end{array}$ \\
\hline 2 ans & $\begin{array}{ll}\mathrm{p} & 3 \\
\mathrm{p} & 1 \\
\mathrm{p} & 1\end{array}$ & $\begin{array}{ll}\text { np } & 9 \\
\text { p } & 6 \\
\text { p } & 7\end{array}$ \\
\hline
\end{tabular}

qualifie de « non précoupés ») soient en fait fortement infestés par la cécidomyie. Il ne faut donc pas compter sur un éventuel manque de coïncidence pour lutter contre $C$. medicaginis.

Le travail du sol pourrait être une seconde solution puisque la nymphose de la cécidomyie est superficielle. Certains producteurs ont donc pratiqué le binage entre les rangs pour tenter de détruire une partie des nymphes. Il n'a jamais été fait d'expérimentation en France pour définir l'intérêt de cette pratique, qui semble tout de même limité dans la mesure où les risques d'infestation en provenance des champs du voisinage existent, des cultures mal entretenues pouvant servir de véritables réservoirs. Nous avons pu constater l'infestation extrêmement importante de champs semés au printemps de l'année d'observation, dont le sol ne peut donc pas contenir de cocons de cécidomyies hivernantes et qui ne sont proches d'aucun champ de luzerne dans un rayon de $300 \mathrm{~m}$.

\section{B. Le traitement du sol par les insecticides}

Des expérimentations infructueuses ont été conduites à ce sujet en France. Les produits organochlorés classiques incorporés au sol des luzernières ont eu, en leur temps, des actions secondaires sur l'entomofaune utile, impliquant des déséquilibres qui ont notamment permis la pullulation de mollusques par suppression de leurs prédateurs (TROUILLON, 1977).

Quant aux autres types de produits, systémiques et très persistants qui servent actuellement à la protection des semis de cultures, leur utilisation est liée généralement à leur localisation et surtout à leur incorporation au sol. Dans le cas de la luzerne installée au moins pour 3 années, il serait nécessaire d'envisager une utilisation en surface, en plein sol, mais arec les matières actives actuellement disponibles, ceci est exclu.

D'ailleurs, en sus des bouleversements dans l'équilibre de la faune du sol, on sait d'après les travaux nord américains (JOHANSEN, 1979) que des risques de contamination du nectar existent, dont les conséquences risquent d'être graves pour les pollinisateurs. Enfin, dans tous les cas de ces traitements de sol, les recontaminations de la cécidomyie en provenance des champs voisins sont possibles.

\section{Les méthodes de prévision}

La prévision à long terme fondée sur les effectifs de nymphes hivernant dans le sol est très lourde à mettre en place puisqu'elle nécessite des prélèvements de terre et des tris fastidieux. STREBLER (1975) a d'ailleurs signalé que les effectifs dénombrés sont peu représentatifs d'un risque éventuel, car les facteurs biotiques (micro-organismes) agissent sur les populations dans le sol. De plus, la variation de taille et de fécondité des cécidomyies entre diverses générations rend la prévision un peu illusoire.

Les risques d'apparitions massives de cécidomyies ont également été reliés aux facteurs climatiques et notamment aux précipitations. Ces résultats, appliqués dans nos conditions de climat atlantique apparaissent peu convaincants dans la mesure où ils conduisent à la prévision d'un risque généralisé des attaques. Un ajustement entre la prévision des pullulations et les conditions climatiques n'en demeure pas moins souhaitable mais adapté à nos conditions. Cette liaison pourrait d'ailleurs se situer, comme le signale STREBLER (1975), dans la prévision à brève échéance reliant certains facteurs physiques aux conditions d'éclosion et de vol des adultes. Cela nécessite cependant la mise en place d'un réseau qui enregistre les observations sur les pullulations et leurs conditions d'apparition. 


\section{Utilisation de méthodes biologiques}

En ce qui concerne l'utilisation d'éventuels facteurs biologiques de régulation des populations de cécidomyies, on ne dispose d'aucune indication pratique pour la mise en œuvre de méthodes de lutte. Selon STREBLER, ce sont les micro-organismes qui, dans la phase souterraine de la vie de la cécidomyie, sont les plus importants facteurs de régulation des populations. Aucune utilisation pratique de ces germes dont la spécificité est inconnue n'est prévisible à court terme. Enfin, quelques mentions de moindre sensibilité de lignées de luzerne à l'infestation par C. medicaginis sont signalées. Des expérimentations préliminaires pour comparer quelques luzernes provenant d'Europe centrale à une variété française ont été conduites dans des cages où apparaissaient des cécidomyies des fleurs. Seule 1 lignée a montré dans une expérience une moindre contamination (estimée par l'extériorisation des galles) ; ce fait ne s'est cependant pas reproduit dans une répétition (BOURNOvILLE, 1983).

\section{CONCLUSION}

On peut retenir en conclusion de cette étude les très importantes contraintes d'organisation pratique de la lutte contre la cécidomyie des fleurs. Le végétal est réceptif durant une longue période à la ponte d'un diptère à vie brève mais dont les générations sont très étalées. La contamination peut provenir du biotope environnant et la lutte chimique doit éviter les effets secondaires sur des auxiliaires indispensables : les pollinisateurs.

L'approche expérimentale privilégiant les résultats de terrain nous a amenés à écarter certaines solutions valables en théorie mais non réalistes dans la pratique.
Les techniques qui permettent de déterminer la présence et l'abondance des adultes de cécidomyies sont lourdes à mettre en œuvre et peu fiables. Dans l'état actuel de l'encadrement de la production de semences de luzerne, on ne dispose d'ailleurs pas des moyens techniques et humains qui permettraient la diffusion immédiate d'ordres de traitement. Il faudrait en effet disposer d'un réseau dense enregistrant sans délai les augmentations brutales d'effectifs des adultes de l'insecte. Enfin, pour l'organisation d'un éventuel réseau de piégeage, il paraît bien difficile d'établir un seuil d'intervention fondé sur les captures. Divers niveaux essayés de ce point de vue à titre expérimental n'ont pas donné satisfaction.

En revanche, à condition de bien repérer le début du bourgeonnement, ce qui implique des observations au niveau de la parcelle, l'époque des interventions peut être fixée de façon très efficace. La possibilité d'utiliser certains pyréthrinoïdes peu toxiques pour les pollinisateurs favorise la réussite de ce système d'intervention qui est vulgarisé pour la lutte contre $C$. medicaginis. Sa simplification pourrait consister à fournir au producteur le délai, sous forme d'une somme de températures, séparant le début de la repousse du stade propice au traitement, ce qui éviterait l'observation de l'état des tiges (HACQUET, 1984).

Reçu le 28 mai 1985. Accepté le 13 décembre 1985.

\section{REMERCIEMENTS}

Nous remercions sincèrement tous nos collègues, ingénieurs régionaux de la FNAMS, ingénieurs du Service de la Protection des végétaux (S.P.V.) des circonscriptions phytosanitaires de PoitouCharentes et Midi-Pyrénées ainsi que les stagiaires qui ont collaboré à la réalisation de ce programme.

\section{RÉFÉRENCES BIBLIOGRAPHIQUES}

Bournoville R., 1983. Etat des connaissances acquises sur les ravageurs de la luzerne en France pour contribuer à la protection intégrée de la culture. In : «C. R. $4^{e}$ Coll. bulgare-français sur la lutte biologique et intégrée contre les ravageurs des grandes cultures ». Acad. Agric. R.P. Bulgarie - Institut de Protection des Plantes, 110-122.

Bournoville R., Bourdoncle B., 1978. Influence des insectes nuisibles sur la production grainière de luzerne dans le département du Tarn. In : Nouvelles des Fourrages à l'I.N.R.A. - étude $\mathrm{n}^{\circ} 63$, S.E.I.-I.N.R.A. ed., 223-227.

Bournoville R., Dontchev K., Sedivy J., 1984. Les ravageurs de la production de graines de luzerne en Europe. C. R. Réunion Elcarpia, Groupe Medicago sativa, Brno, OSEVA ed. Troubsko, 359-364.

Cairaschi E. A., 1973. Quelques recommandations pour la protection des cultures de luzernes-graines. Phytoma, 244, 9-12.

Coutin R., 1962. La lutte contre la cécidomyie (Contarinia medicaginis) des fleurs de luzerne. Rev. Zool. agric. Pathol. veg., 61, 8-22.
Debray Ph., Leblanc J., Tasei J. N., Onillon H., 1984. Evaluation de la toxicité pratique de la sumicidin sur l'abeille domestique (Apis mellifera $\mathrm{L}$.) et l'abeille solitaire (Megachile rotundata $\mathrm{F}$.) en activité sur colza et luzerne en fleurs. Les Colloques de l'I.N.R.A. $n^{\circ} 21$ (I.N.R.A., éd. Paris), 163-168.

Hacquet J., 1984. Luzerne porte-graine : comment lutter contre la cécidomyie des fleurs. Bull. semences, 87, 25-28.

Johansen C. A., 1979. Honeybee poisoning by chemicals : signs, contributing factors, current problems and prevention. Bee World, 60, 109-127.

Strebler G., 1968. Etude d'un Itonidide cécidogène (Contarinia medicaginis Kief.) et de son hôte. Marcellia, 35, 3, 155-188.

Strebler G., 1975. La prévision des pullulations des cécidomyies nuisibles à la production de semences de luzerne. Exemple de Contarinia medicaginis Kief. Rev. Zool. agric. Pathol. veg., 74, 1-35.

Trouillon L., 1977. Commentaires sur une méthode de lutte contre des ravageurs de luzerne, in : C. $R$. $5^{e}$ journées de Phytiatrie et de Phytopharmacie circum-méditerranéennes, Rabat, 348-350. 